\title{
Article \\ Genomic Identification and Functional Analysis of JHAMTs in the Pond Wolf Spider, Pardosa pseudoannulata
}

\author{
Zhi-Ming Yang, Yong Wu, Fang-Fang Li, Zhang-Jin Zhou, Na Yu and Ze-Wen Liu *
}

check for updates

Citation: Yang, Z.-M.; Wu, Y.; Li, F.-F.; Zhou, Z.-J.; Yu, N.; Liu, Z.-W. Genomic Identification and Functional Analysis of JHAMTs in the Pond Wolf Spider, Pardosa pseudoannulata. Int. J. Mol. Sci. 2021, 22, 11721. https:// doi.org/10.3390/ijms222111721

Academic Editor: Steve Peigneur

Received: 14 September 2021

Accepted: 26 October 2021

Published: 29 October 2021

Publisher's Note: MDPI stays neutral with regard to jurisdictional claims in published maps and institutional affiliations.

Copyright: (c) 2021 by the authors. Licensee MDPI, Basel, Switzerland. This article is an open access article distributed under the terms and conditions of the Creative Commons Attribution (CC BY) license (https:// creativecommons.org/licenses/by/ $4.0 /)$.
Key Laboratory of Integrated Management of Crop Diseases and Pests (Ministry of Education), College of Plant Protection, Nanjing Agricultural University, Nanjing 210095, China; 2018202054@njau.edu.cn (Z.-M.Y.); 2020102122@stu.njau.edu.cn (Y.W.); 2019102137@njau.edu.cn (F.-F.L.); 2020102124@stu.njau.edu.cn (Z.-J.Z.); yuna@njau.edu.cn (N.Y.)

* Correspondence: liuzewen@njau.edu.cn; Tel./Fax: +86-25-8439-9051

\begin{abstract}
Juvenile hormone (JH) plays a critical role in many physiological activities of Arthropoda. Juvenile hormone acid methyltransferase (JHAMT) is involved in the last steps of JH biosynthesis as an important rate-limiting enzyme. In recent studies, an increasing number of JHAMTs were identified in arthropods, but no JHAMT was reported in spiders. Herein, eight JHAMTs were identified in the pond wolf spider, Pardosa pseudoannulata, all containing the well conserved S-adenosyl-L-methionine binding motif. JHAMT-1 and the other seven JHAMTs were located at chromosome 13 and chromosome 1, respectively. Multiple alignment and phylogenetic analysis showed that JHAMT-1 was grouped together with insect JHAMTs independently and shared high similarities with insect JHAMTs compared to the other seven JHAMTs. In addition, JHAMT-1, JHAMT-2, and JHAMT-3 were highly expressed in the abdomen of spiderlings and could respond to the stimulation of exogenous farnesoic acid. Meanwhile, knockdown of these three JHAMTs caused the overweight and accelerated molting of spiderlings. These results demonstrated the cooperation of multi-JHAMTs in spider development and provided a new evolutionary perspective of the expansion of JHAMT in Arachnida.
\end{abstract}

Keywords: Pardosa pseudoannulata; JHAMT; phylogeny; RNA interference; gene expansion

\section{Introduction}

Juvenile hormone $(\mathrm{JH})$, the sesquiterpenoid hormone, regulates development, metamorphosis, reproduction, and diapause in Arthropoda [1-6]. Juvenile hormone acid methyltransferase (JHAMT) is a highly specific rate-limiting enzyme in JH biosynthesis in insects [7]. It belongs to the family of S-adenosyl-L-methionine (SAM)-dependent methyltransferases (MTs) which transfer a methyl group from methyl donor SAM to the carboxyl group of substrates $[7,8]$. JHAMT catalyzes the methylation in the last steps of juvenile hormone III (JH III) biosynthesis, converting farnesoic acid (FA) to methyl farnesoate (MF) or converting JH III acid (JHA III) to JH III [1,7].

Since the first JHAMT was identified in Bombyx mori [7], a great number of orthologs of JHAMT have been characterized in insects, such as Drosophila melanogaster [9], Tribolium castaneum [10], Aedes aegypti [11], Acyrthosiphon pisum [12], Apis mellifera [13], Leptinotarsa decemlineata [14], Blattella germanica [15], and so on. Silencing JHAMT lowered JH titer [14], caused larval precocious metamorphosis [10], and inhibited vitellogenin synthesis [15]. Taken together, JHAMT was highly correlated to JH biosynthesis and played essential roles in metamorphosis and reproduction in insects.

Recently, with the developments of the deep sequenced genomes and transcriptomes, JHAMT orthologs were also identified in Arachnida, including mite Tetranychus urticae [16,17], scorpion Mesobuthus martensii [18], ticks Ixodes scapularis [19,20], Dermacentor variabilis [20], and Ornithodoros turicata [20]. However, no study related to the identification and function of JHAMT in JH biosynthesis was reported in spiders. In this study, we took advantage 
of the genomic and transcriptomic databases to identify and characterize JHAMTs in the pond wolf spider Pardosa pseudoannulata. In addition, the function of JHAMTs in spider development was demonstrated by RNA interference (RNAi).

\section{Results}

\subsection{Characterization of JHAMTs}

Eight full-length JHAMTs were identified in P. pseudoannulata, namely, JHAMT-1, -2, -3, $-4,-5,-6,-7$, and -8 , and submitted to GenBank (GenBank accession number: MZ321024, MZ321025, MZ321026, MZ321027, MZ321028, MZ321029, MZ321030, and MZ321031, respectively). Their open reading frames (ORFs) were 822 base pairs (bp), $816 \mathrm{bp}, 825 \mathrm{bp}$, $810 \mathrm{bp}, 819 \mathrm{bp}, 855 \mathrm{bp}, 822 \mathrm{bp}$, and $861 \mathrm{bp}$ which encoded a protein of 273 amino acids (aa), 271 aa, 274 aa, 269 aa, 272 aa, 284 aa, 273 aa, and 286 aa, respectively. The accuracy of the complete sequence was confirmed by PCR (Figure S1). Eight JHAMTs were located at two chromosomes with JHAMT-1 at chromosome 13 and the rests at chromosome 1 (Figure 1). The predicted amino acid sequences of eight JHAMTs contained the well conserved SAMbinding motif (motif I) and their secondary structure incorporated the alternation of nine $\alpha$-helices and six $\beta$-strands comparing with the typical core fold of SAM-MTs (Figure 2).

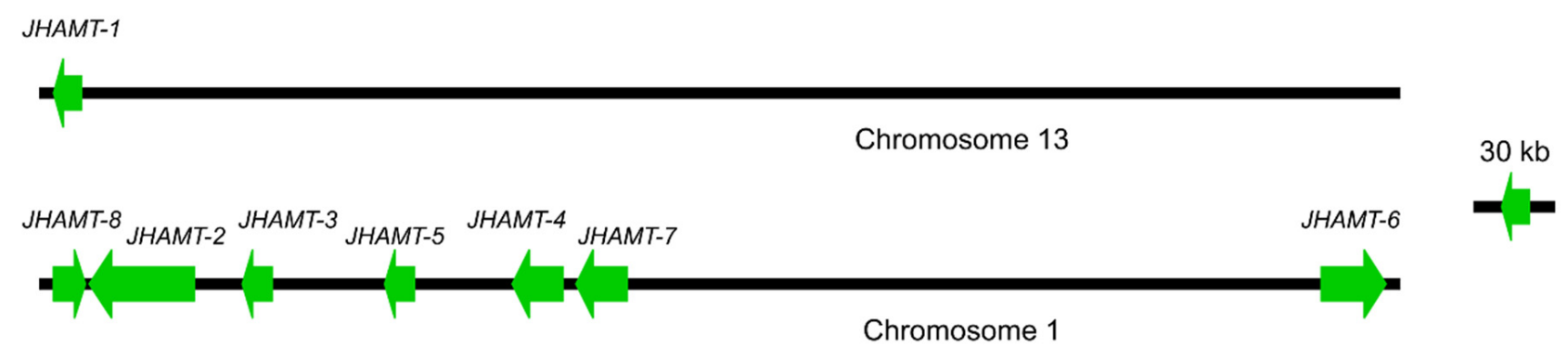

Figure 1. Chromosomal distribution of eight JHAMT genes in P. pseudoannulata. Only regions (black line) containing target genes were illustrated at the chromosomes. Green arrows represented genes and indicated the relative direction of genes to the chromosome.

\subsection{Phylogenetic Analysis}

The numbers of 3, 2, 6, 1, 1, and 20 JHAMT genes were identified from genomes of three spiders $P$. tepidariorum, S. mimosarum, and T. clavipes, two mites $V$. destructor and G. occidentalis, and a scorpion C. sculpturatus, respectively (Figure 3A, Table S2). It was very difficult to strict distinguish the JHAMTs between arachnids and insects according to the phylogenetic analysis of JHAMTs because one of the multi-JHAMT copies from arachnids was grouped together with those in insects and the remaining arachnid JHAMTs were grouped together (Figure 3A). Meanwhile, there were differences in similarities of amino acid sequences between eight JHAMTs from P. pseudoannulata and four JHAMTs from insects (Figure 3B). In P. pseudoannulata, JHAMT-1 shared the similarities of $24 \%, 23 \%$, $26 \%, 27 \%, 24 \%, 25 \%$, and $26 \%$ with JHAMT-2, $-3,-4,-5,-6,-7$, and -8 , respectively, which were lower than that of four JHAMTs from insects with over $32 \%$ similarities. Among the other seven JHAMTs from $P$. pseudoannulata, the similarities were $57-72 \%$ among JHAMT-2, $-3,-4$, and -5 , and $75-79 \%$ among JHAMT- $6,-7$, and -8 , and $39-46 \%$ between these two groups (Figure 3B). 

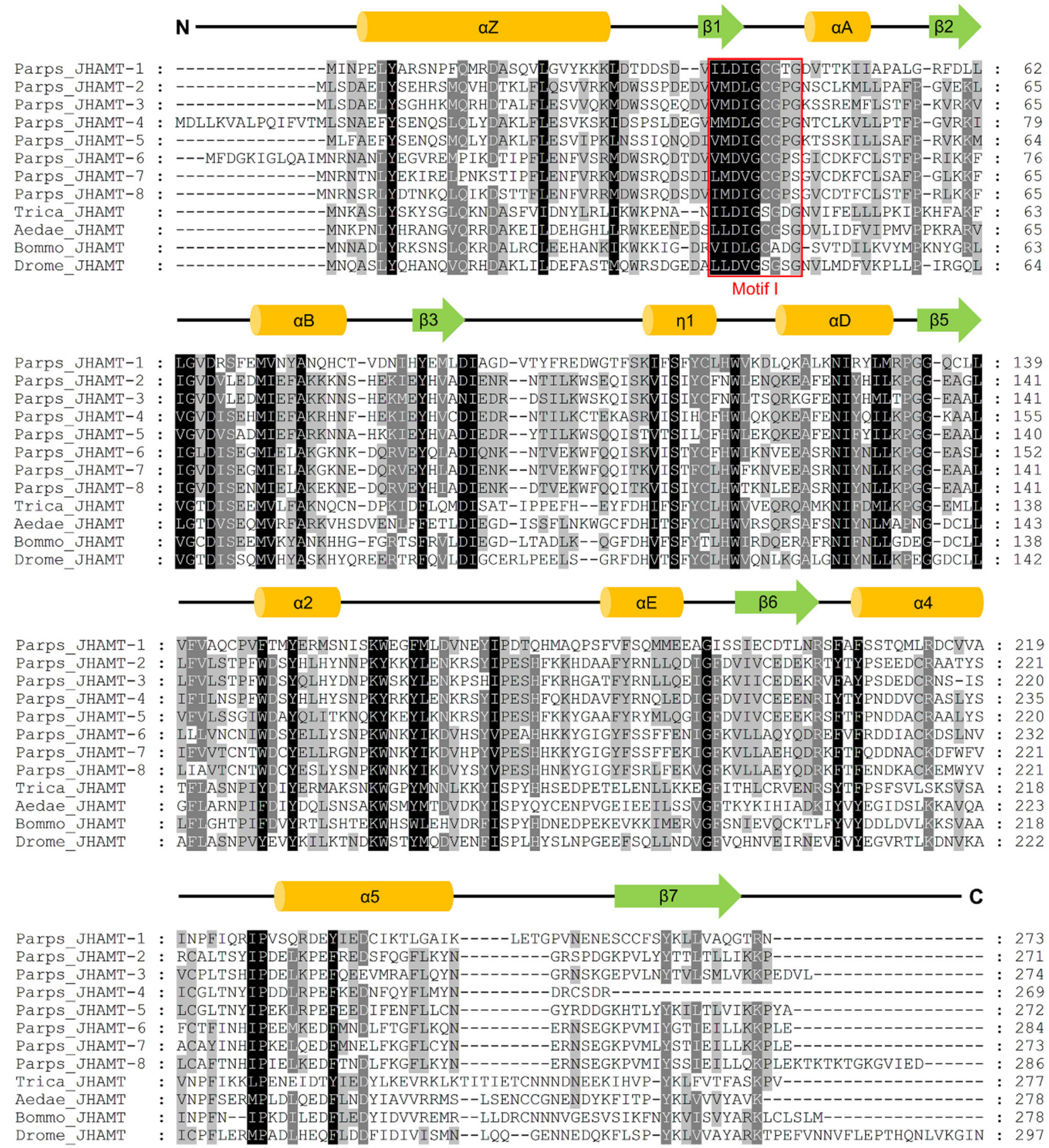

Figure 2. Conserved regions of JHAMTs from P. pseudoannulata and insects. Residues highlighted by red box were the conserved S-adenosyl-L-methionine binding motif (motif I). Secondary structural elements of JHAMTs were showed as orange cylinders ( $\alpha$-helices) and green arrows ( $\beta$-strands) marked above the sequences. Parps, Pardosa pseudoannulata; Trica, Tribolium castaneum; Aedae, Aedes aegypti; Bommo, Bombyx mori; Drome, Drosophila melanogaster. 
A

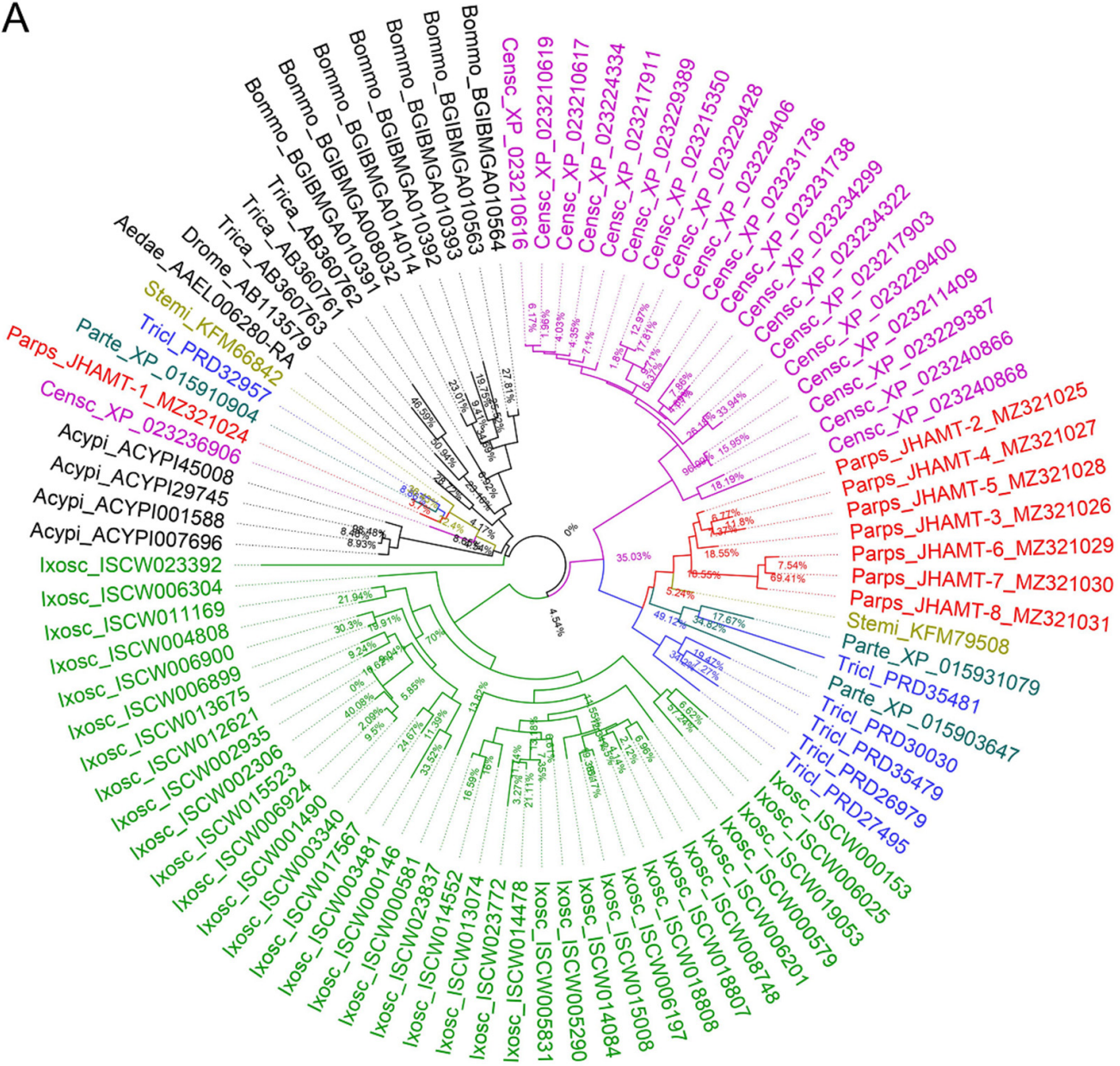

B

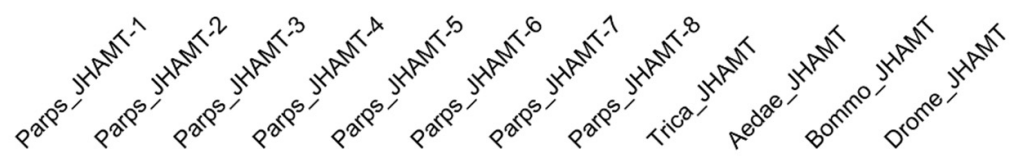

Parps_JHAMT-1

Parps_JHAMT-2

Parps_JHAMT-3

Parps_JHAMT-4

Parps_JHAMT-5

Parps_JHAMT-6

Parps_JHAMT-7

Parps_JHAMT-8

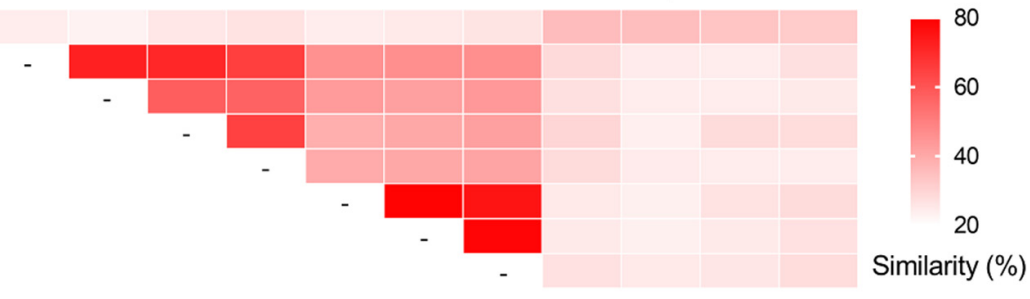

Figure 3. Phylogenetic analysis (A) and similarities (B) of JHAMTs from arachnids and insects. These JHAMTs were retrieved from the previous reports [9-11,19,21,22] or identified in the present study (Table S2). The gene ID was represented by the abbreviation of the Latin name of the species \& its accession number. The phylogenetic tree was constructed by IQ-TREE and processed in Figtree software. The black JHAMTs were insects. The JHAMTs from an arachnid species were marked with the same color. The numbers at base of nodes were the branch times. The heatmap of similarities was processed in GraphPad Prism 7. Bommo, Bombyx mori; Aedae, Aedes aegypti; Trica, Tribolium castaneum; Drome, Drosophila melanogaster; Acypi, Acyrthosiphon pisum; Ixosc, Ixodes scapularis; Parps, Pardosa pseudoannulata; Parte, Parasteatoda tepidariorum; Stemi, Stegodyphus mimosarum; Tricl, Trichonephila clavipes; Censc, Centruroides sculpturatus.

\subsection{Spatiotemporal Expression Profile}

There were different expression profiles of eight JHAMTs in four developmental stages, egg, the second-instar spiderling, adult female and adult male (Figure 4A). JHAMT-1 was expressed higher in males and lower in eggs. JHAMT-2 was significantly expressed in 
spiderlings and males rather than eggs and females. JHAMT-3 and JHAMT-4 showed the opposite expression patterns with JHAMT-3 highly expressed in spiderlings and adults, while JHAMT-4 highly expressed in eggs. Both JHAMT-5 and JHAMT-8 showed low expression levels in the four samples. Both JHAMT-6 and JHAMT-7 were significantly expressed in males, especially JHAMT-6 (Figure 4A). The spiderlings were separated into two parts, cephalothorax and abdomen (Figure 4B). JHAMT-1, JHAMT-2, JHAMT-3, and JHAMT-6 were significantly expressed in abdomen. Both JHAMT-5 and JHAMT-8 were significantly expressed in cephalothorax. There was no expressional difference of JHAMT-4 and JHAMT-7 between the two parts (Figure 4B). Spatial expressions of eight JHAMTs showed the specific patterns in six tissues, brain, venom gland, fat body, intestine, ovary, and testes (Figure 4C). JHAMT-1 was highly expressed in brains, venom glands, and testes. JHAMT-2 was significantly expressed in fat bodies and intestines. Both JHAMT-3 and JHAMT-6 showed significant expressions in fat bodies. Both JHAMT-4 and JHAMT-5 showed remarkable expressions in venom glands and ovaries, and traces in the rest tissues. Both JHAMT-7 and JHAMT-8 showed notable expressions in venom glands, especially JHAMT-8 (Figure 4C).

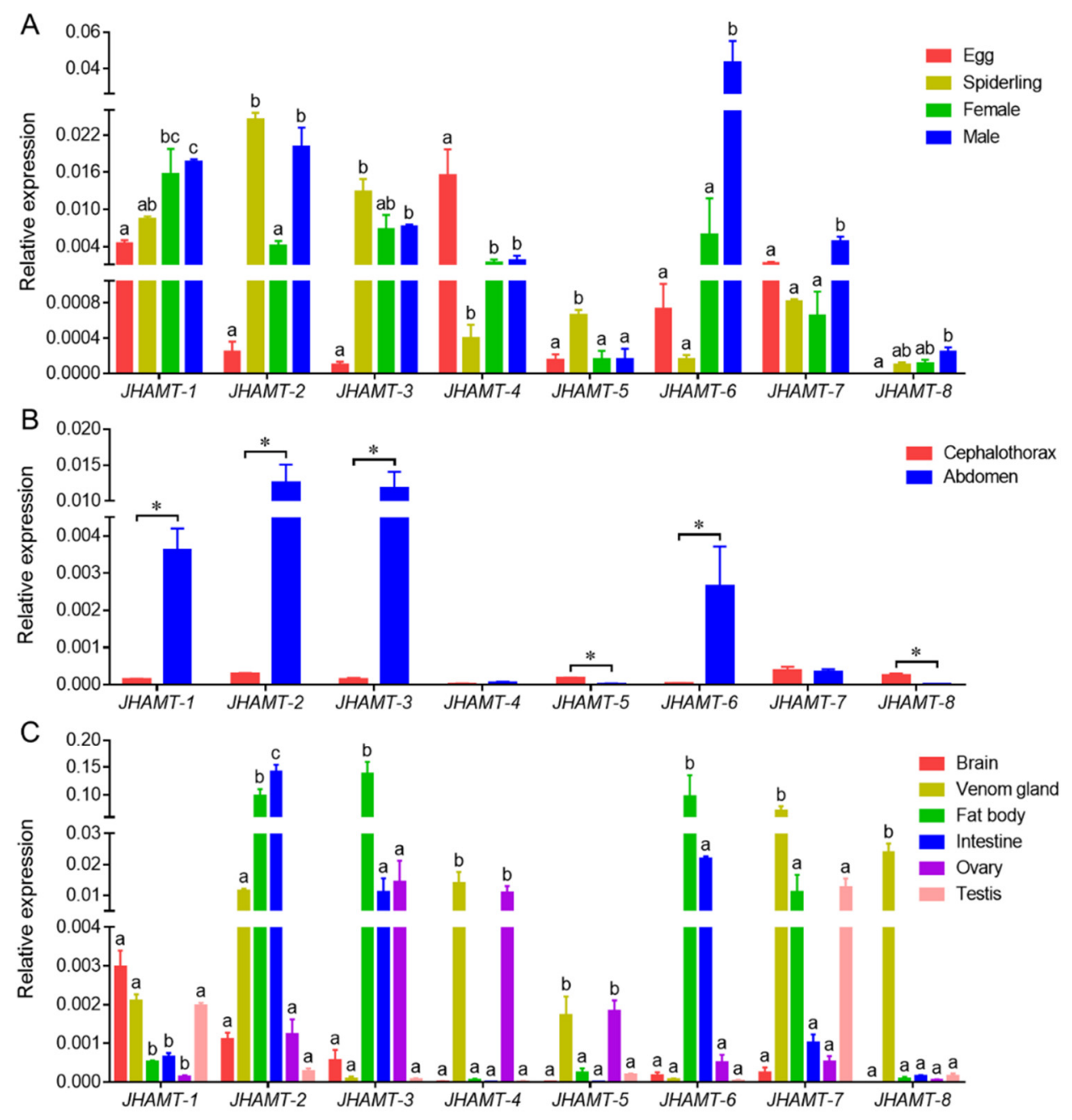

Figure 4. Spatiotemporal expression profiles of eight JHAMT genes in four developmental stages (egg, the second-instar spiderling, adult female, and adult male) (A), two parts (cephalothorax and abdomen) from the second-instar spiderlings (B), and six tissues (brain, venom gland, fat body, intestine, ovary, and testes) from adult females and males (C). Different lower-case letters ( $a, b$, or c) indicated the significant difference of gene expression in four developmental samples and in six tissular samples at $p<0.05$. ${ }^{*}, p<0.05$. 


\subsection{Effect of Farnesoic Acid Administration}

To determine the function of eight JHAMTs in JH synthesis, the changes of JHAMT transcriptional level were detected in spiderlings treated by FA. The transcriptional level of JHAMT-1 was significantly downregulated after FA treatment, but both JHAMT-2 and JHAMT-3 were significantly stimulated (Figure 5). In addition, the other 5 JHAMTs showed no difference between FA treatment and control group (Figure 5).

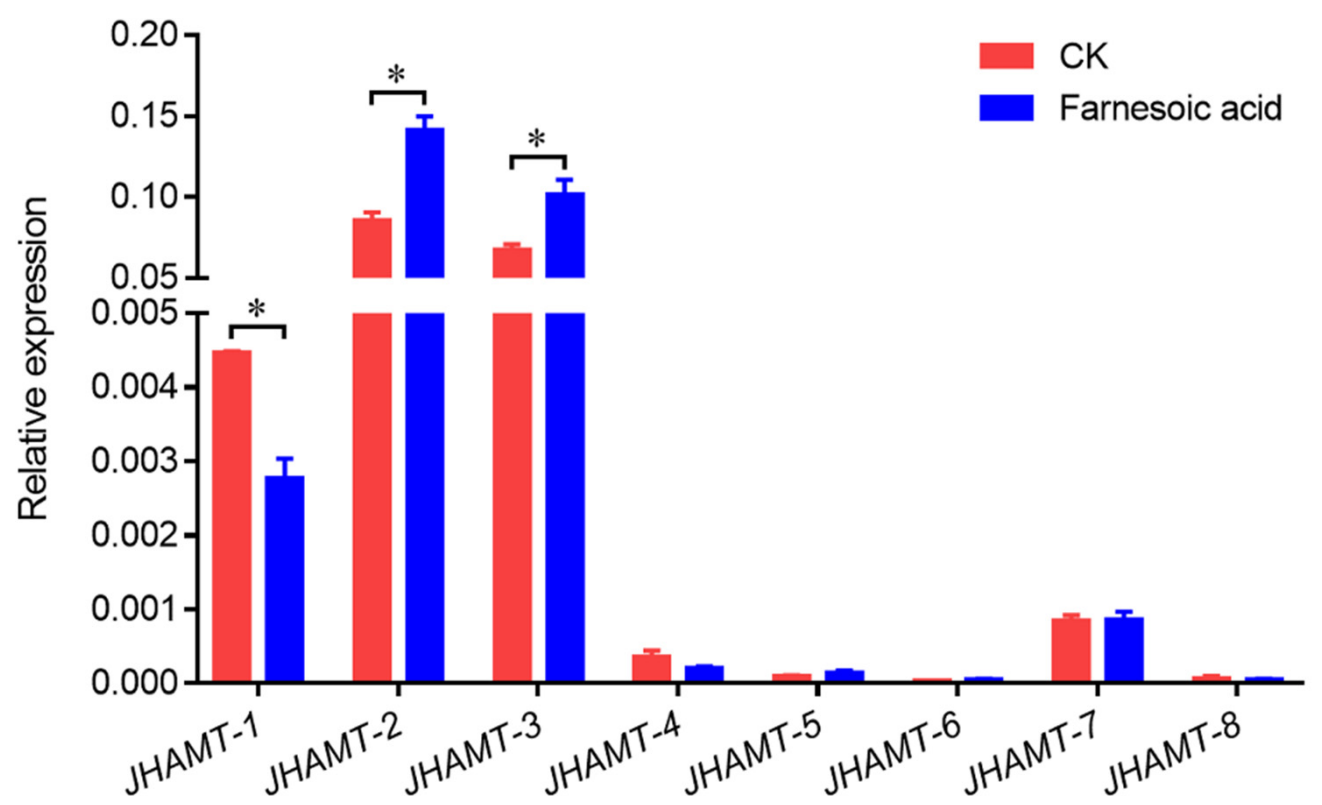

Figure 5. Relative expressions of eight JHAMT genes in farnesoic acid-applied the second-instar spiderlings. CK, control group containing 200 time-diluted ethanol. ${ }^{*}, p<0.05$.

\subsection{Effect of JHAMT Silencing}

Compared with dseGFP-treated spiderlings, JHAMT-1, JHAMT-2, and JHAMT-3 were downregulated by $47 \%$ (Figure $6 \mathrm{~A}$ ), 65\% (Figure 6B), and 65\% (Figure 6C) in target dsJHAMT-treated spiderlings, respectively. The expressions of the other non-target JHAMTs were not changed in dsJHAMT treatments except for the increase of JHAMT-1 expression in dsJHAMT-3-treated spiderlings (Figure 6C). The spiderlings of both control and treatment groups were weighted at $72 \mathrm{~h}$ after injection. The average weight of dseGFP, dsJHAMT-1, dsJHAMT-2, and dsJHAMT-3-treated spiderlings were $0.63 \mathrm{mg}, 0.74 \mathrm{mg}$, $0.76 \mathrm{mg}$, and $0.71 \mathrm{mg}$, respectively (Figure 6D). The spiderlings in the three dsJHAMT treatment groups were weighted heavier than dseGFP group. In addition, the molting rates of spiderlings were $48 \%, 71 \%, 63 \%$, and $57 \%$ at $96 \mathrm{~h}$ in dseGFP, dsJHAMT-1, dsJHAMT-2, and dsJHAMT-3 groups, respectively (Figure $6 \mathrm{E}$ ), and at $120 \mathrm{~h}$, they were $64 \%, 87 \%, 76 \%$, and $72 \%$, respectively (Figure $6 \mathrm{~F}$ ). The molting rates of the three dsJHAMT treatment groups had statistical difference with dseGFP group except for dsJHAMT-3 group in both counts. 

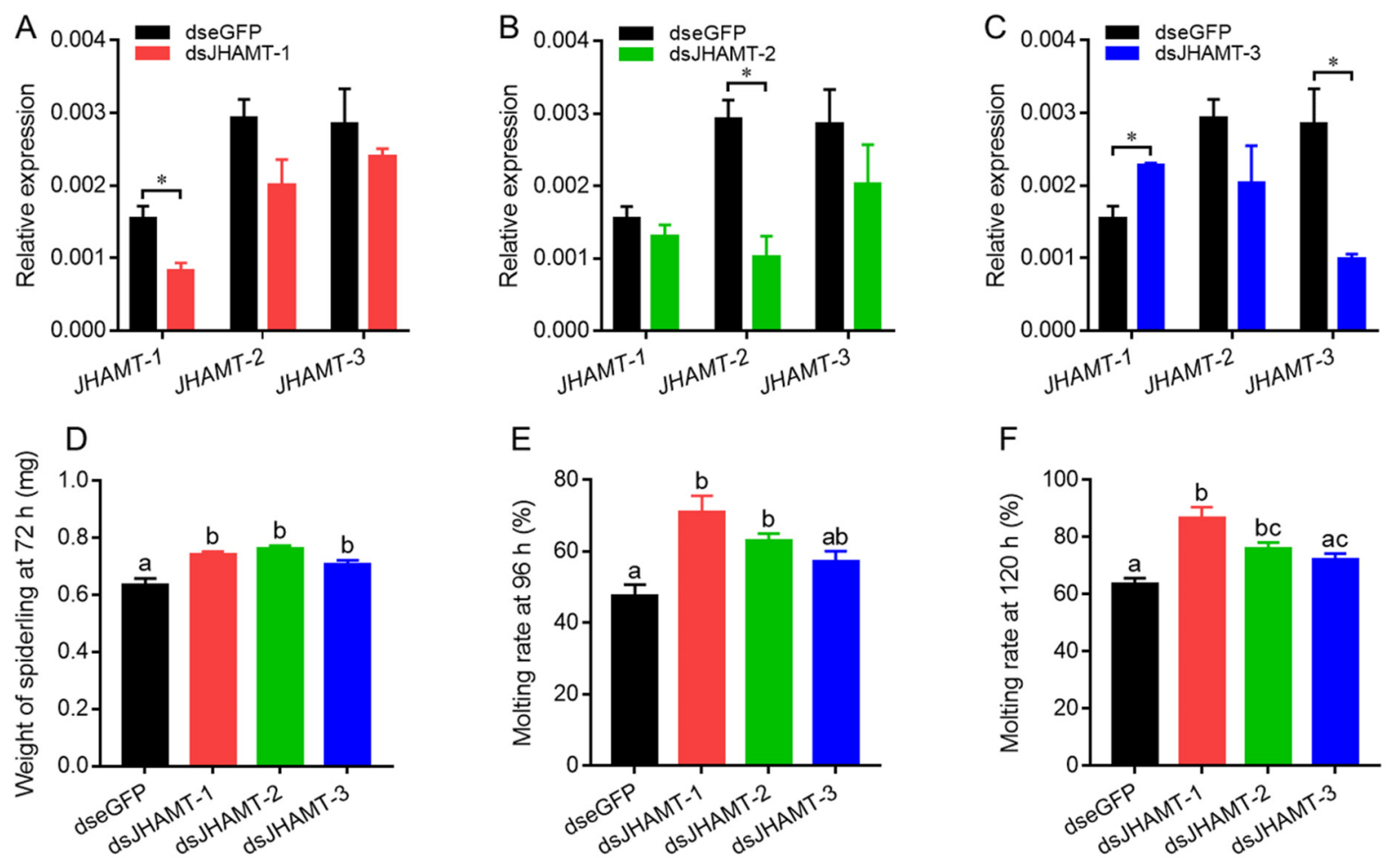

Figure 6. Effects of injection of dsJHAMT-1, dsJHAMT-2, and dsJHAMT-3 in the second-instar spiderlings. Relative expressions of JHAMT-1, JHAMT-2, and JHAMT-3 in spiderlings treated by dsJHAMT-1 (A), dsJHAMT-2 (B), and dsJHAMT3 (C). Weight of spiderlings at $72 \mathrm{~h}(\mathbf{D})$ and molting rates of spiderlings at $96 \mathrm{~h}(\mathrm{E})$ and $120 \mathrm{~h}(\mathrm{~F})$ after injection of dseGFP and three dsJHAMT. Different lower-case letters $(a, b$, or $c)$ indicated the significant difference of weight and molting rate of spiderlings between dseGFP and three dsJHAMT groups at $p<0.05 .{ }^{*}, p<0.05$.

\section{Discussion}

In the present study, eight JHAMTs were identified in the whole genome of P. pseudoannulata. It was the first detailed characterization of JHMAT in spiders. Similarly, multi-copies of JHAMT were also found in several arachnids, including three spiders P. tepidariorum, S. mimosarum, and T. clavipes, and a scorpion C. sculpturatus. Moreover, the numbers of 3 and 44 JHAMT genes have been reported in the genomic works of scorpion M. martensii [18] and tick I. scapularis [19], respectively. However, the same with T. urticae [16,17], only one JHAMT presented in two mite species, $V$. destructor and G. occidentalis. Therefore, there was the striking expansion of JHAMT in spiders, scorpions, and ticks, but not in mites. The duplication of JHAMT also occurred in insects, including T. castaneum [10], A. pisum [12], and B. mori [21], although only one JHAMT in most insect species. Interesting results from the phylogenetic tree showed that there was always a JHAMT in arachnids that was independent of the other copies and grouped together with that of insects. Further, JHAMT-1 from P. pseudoannulata showed high similarities of amino acid sequences with insect JHAMTs than the other seven JHAMTs. In the chromosomal distribution of eight JHAMT genes, JHAMT-1 was located at chromosome 13 and the remaining JHAMTs were located together at chromosome 1 . From the above results, we speculated that an ancestral JHAMT gene was presented in both Arachnida and Insecta, and the new JHAMTs were developed and duplicated in arachnids after the separation of insects. In the future, more evidence related genomic analyses of arachnid species are needed to confirm this hypothesis.

JHAMT, as the key rate-limiting enzyme in regulation of JH titer, is involved in the last steps of the active $\mathrm{JH}$ product biosynthesis pathway in insects by transferring the methyl group of SAM to the carboxyl group of FA or JHA III [7]. Differing from corpora allata as the main biosynthetic site of JH III in insects [23], these JH biosynthesis-related genes were highly expressed in abdomen of P. pseudoannulata [24]. It indicated that JHAMT-1, JHAMT-2, 
JHAMT-3, and JAHMT-6 were involved in JH biosynthesis in P. pseudoannulata due to their specific expressions in abdomen. Just as predicted, the gene expressions of JHAMT-1, JHAMT-2, and JAHMT-3 were significantly changed by exogenous FA application, but not JHAMT-6. JHAMT-2 and JHAMT-3 were stimulated by FA, but opposite in JHAMT-1. It might be that there was a cooperative mechanism between the JHAMTs to respond to the changes of FA. In addition, some JHAMTs might have functional differentiation, such as JHAMT-7 and JHAMT-8 involved in toxin production in the spider because of their high expressions in venom glands.

The development of spiderlings was affected by JHAMT silencing. JHAMT downregulation could increase the weight of spiderlings. Meanwhile, the accelerated molting was embodied in the higher molting rate of dsJHAMT treatments than that of control group at both $96 \mathrm{~h}$ and $120 \mathrm{~h}$. However, JHAMT-3 had no effects in spiderling's development although there was a statistical difference in the weight of spiderlings, possibly due to the functional absence of JHAMT-3, which was rescued by the increased expression of JHAMT-1 in dsJHAMT-3-treated spiderlings. To sum up, JHAMTs regulate spider development in a coordinated way.

\section{Materials and Methods}

\subsection{Identification and Characterization of JHAMTs}

The putative JHAMTs were retrieved from the protein database predicated from the chromosome-level genome of P. pseudoannulata (GenBank accession number: JAGEOH000000000) using the orthologs from B. mori [7], D. melanogaster [9], T. castaneum [10], and A. aegypti [11] (GenBank accession number: BAC98835, BAC98836, BAG30999, and EAT42177, respectively) as query proteins via the command-line tool Exonerate (version 2.2, EMBL-EBI, Cambridge, UK) [25]. The obtained JHAMT sequences were confirmed by the nine transcriptomes of P. pseudoannulata (GenBank accession number: SRR8083389-SRR8083396, and SRR8083398, respectively) and manually corrected gap prediction and UTR to get the complete ORF by multiple alignment in Clustal $X$ (version 2.1, University College Dublin, Dublin, Ireland) [26]. The complete ORF of JHAMT was confirmed by PCR. The specific primers were designed using Beacon Designer (version 7.92, PREMIER Biosoft, San Francisco, CA, USA) (Table S1) and synthesized by Genscript (Genscript, Nanjing, China). The PCR products were sequenced in Tsingke (Tsingke Biotechnology, Nanjing, China). The putative JHAMTs were also surveyed in six arachnids which had genomic database entries using the same methods, including Parasteatoda tepidariorum, Stegodyphus mimosarum, Trichonephila clavipes, Varroa destructor, Galendromus occidentalis, and Centruroides sculpturatus (downloaded from https:/ / www.ncbi. nlm.nih.gov/genome/, the genomic database of T. clavipes last accessed on 18 October 2019, the rest were last accessed on 18 March 2021). Multiple alignment was performed by Clustal X [26] and illustrated in GENEDOC (version 2.7, downloaded from https:// genedoc.software.informer.com/download/, accessed on 29 March 2016) [27]. The maximum likelihood phylogenetic tree was constructed by IQ-TREE (version 2.1.3, downloaded from http: / / www.iqtree.org/, accessed on 22 October 2021) [28] and processed in Figtree (version 1.4.3, downloaded from https:/ / github.com/rambaut/figtree/releases, accessed on 4 October 2016). Secondary structure was derived from the consistent predictions of different tools, including JPred4 (http:/ / www.compbio.dundee.ac.uk/jpred/, accessed on 24 April 2020) [29], PSIPRED (http:/ / bioinf.cs.ucl.ac.uk/psipred/, accessed on 24 April 2020) [30], and PredicProtein (https:/ / www.predictprotein.org/, accessed on 24 April 2020) [31]. The predicted topology of the fold was presented based on previous descriptions $[8,11]$. Gene locations at chromosome were drawn by IBS (version 1.0.3, downloaded from http:/ /ibs.biocuckoo.org/download.php, accessed on 5 June 2018) [32].

\subsection{Spiders}

P. pseudoannulata adults were collected from a rice field in Nanjing (Jiangsu province, China) in May 2020 and housed in $500 \mathrm{~mL}$ plastic cups individually at $28 \pm 1{ }^{\circ} \mathrm{C}$ and 
16/8 h light/dark and fed with Nilaparvata lugens. The spiders were reared in laboratory conditions for at least one month before experiments began. Four developmental samples, egg, the second-instar spiderling, adult female, and adult male, were collected individually and 5-10 eggsacs or spiders were pooled as one sample. Two parts, cephalothorax and abdomen, were dissected from 10 the second-instar spiderlings. Six tissular samples, brain, venom gland, fat body, intestine, ovary, and testes, were dissected from 20 adult females and males. Each sample was carried out with three biological replicates.

\subsection{Farnesoic Acid Treatment}

FA was purchased from Echelon (Salt Lake City, UT, USA) and dissolved in absolute ethanol to get the stock solution of $10 \mathrm{mg} / \mathrm{mL}$. The stock solution was diluted using sterilized water to get the working concentration of $0.05 \mathrm{mg} / \mathrm{mL}$. Ethanol diluted 200 times was set as negative control. The day 1 second-instar spiderlings were starved in petri dishes ( $3.5 \mathrm{~cm}$ in diameter) individually for $12 \mathrm{~h}$ before bioassay. The working solution soaked in absorbent cotton was supplied to each spiderling and solution-cotton was refreshed every $24 \mathrm{~h}$. The spiderlings were fed with a few N. lugens after exposure to working solution for $36 \mathrm{~h}$. Ten spiderlings were harvested at $72 \mathrm{~h}$ to pool as one sample and each sample was carried out with three biological replicates.

\subsection{RNA Interference}

The gene specific primers with extended T7 RNA polymerase promoter sequence on the $5^{\prime}$ end were designed using Beacon Designer (Table S1) and synthesized by Genscript. The gene fragment was amplified using Phanta ${ }^{\circledR}$ Max Super-Fidelity DNA Polymerase (Vazyme, Nanjing, China) and then purified using GeneJET Gel Extraction Kit (Thermo Scientific, Carlsbad, CA, USA) according to the manufacturer's instructions. dsRNA was synthesized using T7 RiboMAX' ${ }^{\mathrm{TM}}$ Express RNAi System (Promega, Madison, WI, USA) according to the manufacturer's instructions. The integrity and quantity of dsRNA were verified by $1.5 \%$ agarose gel electrophoresis and NanoPhotometer spectrophotometer (IMPLEN, Westlake Village, CA, USA) respectively. dsRNA against enhanced green fluorescent protein (eGFP, GenBank accession number: KC896843) was used as the negative control. Delivery of dsRNA by injection have been described in the previous report [33]. This method was used to investigate the biological function of JHMATs in P. pseudoannulata in the present study. Briefly, after anaesthetization by carbon dioxide, the day 1 second-instar spiderlings were kept in an agar gel plate and microinjected with $10 \mathrm{~nL}$ of $50 \mathrm{ng}$ dsRNA individually from the injection site of ventral abdomen. The injected-spiderlings were transferred into petri dishes individually and fed with $N$. lugens. Individuals died of mechanical injury within $12 \mathrm{~h}$ were removed. Injected spiderlings were divided into two groups. Group I was used for quantitative PCR (qPCR). The number of 10 spiderlings of each dsRNA treatment were harvested at $48 \mathrm{~h}$ to pool as one sample. Group II was used for phenotypic responses. The number of 15-20 spiderlings of each dsRNA treatment were used to record the phenotypes. The spiderlings were weighted at $72 \mathrm{~h}$ and the counts of molts were recorded at both $96 \mathrm{~h}$ and $120 \mathrm{~h}$. The experiment was conducted three times independently.

\subsection{Real-Time Quantitative PCR}

Total RNA was extracted using Trizol $^{\mathrm{TM}}$ reagent (Invitrogen, Carlsbad, CA, USA) according to the manufacturer's instructions. The integrity and quantity of RNA were verified by $1.5 \%$ agarose gel electrophoresis and NanoPhotometer spectrophotometer (IMPLEN, Westlake Village, CA, USA) respectively. cDNA was synthesized using PrimeScript RT Reagent Kit (TaKaRa, Kyoto, Japan) according to the manufacturer's instructions. Two reference genes of elongation factor- 1 alpha ( $E F-1 \alpha$, GenBank accession number: KJ888948) and glyceraldehyde-3-phosphatedehydrogenase (GAPDH, GenBank accession number: KJ888949) were selected based on previous description [33]. Primers for qPCR were designed using Beacon Designer (Table S1) and synthesized by Genscript. The specificity 
and efficiency of the primers were verified via melting curve and standard curve assay respectively. The components of qPCR reaction were made using TB Green Premix Ex Taq II Kit (TaKaRa, Kyoto, Japan) according to the manufacturer's instructions and performed on QuantStudio Real-Time PCR System (Applied Biosystems, Foster City, CA, USA). Each reaction was carried out with two technical replicates.

\subsection{Data Analysis}

The relative gene expression was related to the geometric mean of two reference genes by the $2^{-\Delta C T}$ method $[34,35]$. Gene expression, weight, and molting rate of spiderlings were presented as mean \pm SEM from three independent biological replicates. Significant differences were analyzed with t-tests for gene expressions between cephalothorax and abdomen, FA treatments, and dsRNA treatments, and with one-way ANOVA followed by Tukey test for gene expressions in four developmental samples and six tissular samples, and weights and molting rates of spiderlings in dsRNA treatments using GraphPad Prism (version 7, GraphPad Software, San Diego, CA, USA) [36].

Supplementary Materials: The following are available online at https:/ / www.mdpi.com/article/ $10.3390 /$ ijms222111721/s1.

Author Contributions: Conceptualization, Z.-M.Y. and Z.-W.L.; Investigation, Z.-M.Y., Y.W., F.-F.L. and Z.-J.Z.; Visualization, Z.-M.Y.; Writing-original draft, Z.-M.Y.; Writing-review \& editing, N.Y. and Z.-W.L.; Funding acquisition, Z.-W.L. All authors have read and agreed to the published version of the manuscript.

Funding: This research was funded by the National Natural Science Foundation of China $(31772185,31972296)$.

Institutional Review Board Statement: Not applicable.

Informed Consent Statement: Not applicable.

Conflicts of Interest: The authors declare no conflict of interest.

\section{References}

1. Goodman, W.G.; Granger, N.A. The juvenile hormones. In Comprehensive Molecular Insect Science; Gilbert, L.I., Iatrou, K., Gill, S.S., Eds.; Elsevier: Oxford, UK, 2005; Volume 3, pp. 319-408.

2. Applebaum, S.W.; Avisar, E.; Heifetz, Y. Juvenile hormone and locust phase. Arch. Insect Biochem. Physiol. 1997, 35, $375-391$. [CrossRef]

3. Hartfelder, K. Insect juvenile hormone: From "status quo" to high society. Braz. J. Med. Biol. Res. 2000, 33, 157-177. [CrossRef]

4. Wyatt, G.R.; Davey, K.G. Cellular and molecular actions of juvenile hormone II. Roles of juvenile hormone in adult insects. Adv. Insect Physiol. 1996, 26, 1-155.

5. Gilbert, L.I.; Granger, N.A.; Roe, R.M. The juvenile hormones: Historical facts and speculations on future research directions. Insect Biochem. Mol. Biol. 2000, 30, 617-644. [CrossRef]

6. Riddiford, L.M. Cellular and molecular actions of juvenile hormone I. General considerations and premetamorphic actions. Adv. Insect Physiol. 1994, 24, 213-274.

7. Shinoda, T.; Itoyama, K. Juvenile hormone acid methyltransferase: A key regulatory enzyme for insect metamorphosis. Proc. Natl. Acad. Sci. USA 2003, 100, 11986-11991. [CrossRef]

8. Martin, J.L.; McMillan, F.M. SAM (dependent) I AM: The S-adenosylmethionine-dependent methyltransferase fold. Curr. Opin. Struct. Biol. 2002, 12, 783-793. [CrossRef]

9. Niwa, R.; Niimi, T.; Honda, N.; Yoshiyama, M.; Itoyama, K.; Kataoka, H.; Shinoda, T. Juvenile hormone acid O-methyltransferase in Drosophila melanogaster. Insect Biochem. Mol. Biol. 2008, 38, 714-720. [CrossRef]

10. Minakuchi, C.; Namiki, T.; Yoshiyama, M.; Shinoda, T. RNAi-mediated knockdown of juvenile hormone acid O-methyltransferase gene causes precocious metamorphosis in the red flour beetle Tribolium castaneum. FEBS J. 2008, 275, 2919-2931. [CrossRef]

11. Mayoral, J.G.; Nouzova, M.; Yoshiyama, M.; Shinoda, T.; Hernandez-Martinez, S.; Dolghih, E.; Turjanski, A.G.; Roitberg, A.E.; Priestap, H.; Perez, M.; et al. Molecular and functional characterization of a juvenile hormone acid methyltransferase expressed in the corpora allata of mosquitoes. Insect Biochem. Mol. Biol. 2009, 39, 31-37. [CrossRef]

12. Ollivier, M.; Legeai, F.; Rispe, C. Comparative analysis of the Acyrthosiphon pisum genome and expressed sequence tag-based gene sets from other aphid species. Insect Mol. Biol. 2010, 19, 33-45. [CrossRef] 
13. Li, W.; Huang, Z.Y.; Liu, F.; Li, Z.; Yan, L.; Zhang, S.; Chen, S.; Zhong, B.; Su, S. Molecular cloning and characterization of juvenile hormone acid methyltransferase in the honey bee, Apis mellifera, and its differential expression during caste differentiation. PLOS ONE 2013, 8, e68544. [CrossRef]

14. Fu, K.-Y.; Li, Q.; Zhou, L.-T.; Meng, Q.-W.; Lu, F.-G.; Guo, W.-C.; Li, G.-Q. Knockdown of juvenile hormone acid methyl transferase severely affects the performance of Leptinotarsa decemlineata (Say) larvae and adults. Pest Manag. Sci. 2016, 72, 1231-1241. [CrossRef] [PubMed]

15. Dominguez, C.V.; Maestro, J.L. Expression of juvenile hormone acid O-methyltransferase and juvenile hormone synthesis in Blattella germanica. Insect Sci. 2018, 25, 787-796. [CrossRef]

16. Grbic, M.; Van Leeuwen, T.; Clark, R.M.; Rombauts, S.; Rouze, P.; Grbic, V.; Osborne, E.J.; Dermauw, W.; Ngoc, P.C.; Ortego, F.; et al. The genome of Tetranychus urticae reveals herbivorous pest adaptations. Nature 2011, 479, 487-492. [CrossRef] [PubMed]

17. Li, G.; Sun, Q.-Z.; Liu, X.-Y.; Zhang, J.; Dou, W.; Niu, J.-Z.; Wang, J.-J. Expression dynamics of key ecdysteroid and juvenile hormone biosynthesis genes imply a coordinated regulation pattern in the molting process of a spider mite, Tetranychus urticae. Exp. Appl. Acarol. 2019, 78, 361-372. [CrossRef] [PubMed]

18. Cao, Z.; Yu, Y.; Wu, Y.; Hao, P.; Di, Z.; He, Y.; Chen, Z.; Yang, W.; Shen, Z.; He, X.; et al. The genome of Mesobuthus martensii reveals a unique adaptation model of arthropods. Nat. Commun. 2013, 4, 2602. [CrossRef] [PubMed]

19. Gulia-Nuss, M.; Nuss, A.B.; Meyer, J.M.; Sonenshine, D.E.; Roe, R.M.; Waterhouse, R.M.; Sattelle, D.B.; de la Fuente, J.; Ribeiro, J.M.; Megy, K.; et al. Genomic insights into the Ixodes scapularis tick vector of Lyme disease. Nat. Commun. 2016, 7, 10507. [CrossRef]

20. Zhu, J.; Khalil, S.M.; Mitchell, R.D.; Bissinger, B.W.; Egekwu, N.; Sonenshine, D.E.; Roe, R.M. Mevalonate-farnesal biosynthesis in ticks: Comparative synganglion transcriptomics and a new perspective. PLoS ONE 2016, 11, e0141084. [CrossRef]

21. Cheng, D.; Meng, M.; Peng, J.; Qian, W.; Kang, L.; Xia, Q. Genome-wide comparison of genes involved in the biosynthesis, metabolism, and signaling of juvenile hormone between silkworm and other insects. Genet. Mol. Biol. 2014, 37, 444-459. [CrossRef] [PubMed]

22. Ishikawa, A.; Ogawa, K.; Gotoh, H.; Walsh, T.K.; Tagu, D.; Brisson, J.A.; Rispe, C.; Jaubert-Possamai, S.; Kanbe, T.; Tsubota, T.; et al. Juvenile hormone titre and related gene expression during the change of reproductive modes in the pea aphid. Insect Mol. Biol. 2012, 21, 49-60. [CrossRef]

23. Tobe, S.S.; Stay, B. Structure and regulation of the corpus allatum. Adv. Insect Physiol. 1985, 18, 305-432.

24. Yang, Z.-M.; Wu, Y.; Xu, G.-M.; Yu, N.; Liu, Z.-W. Methyl farnesoate acts as the endogenous juvenile hormone in spider moulting. Insect Mol. Biol. 2021, submitted.

25. Slater, G.S.; Birney, E. Automated generation of heuristics for biological sequence comparison. BMC Bioinform. 2005, 6, 31. [CrossRef]

26. Larkin, M.A.; Blackshields, G.; Brown, N.P.; Chenna, R.; McGettigan, P.A.; McWilliam, H.; Valentin, F.; Wallace, I.M.; Wilm, A.; Lopez, R.; et al. Clustal W and Clustal X version 2.0. Bioinformatics 2007, 23, 2947-2948. [CrossRef]

27. Nicholas, K.B.; Nicholas, H.B., Jr.; Deerfield, D.W., II. GeneDoc: Analysis and visualization of genetic variation. EMBNEW News $1997,4,14$.

28. Nguyen, L.T.; Schmidt, H.A.; Von Haeseler, A.; Minh, B.Q. IQ-TREE: A fast and effective stochastic algorithm for estimating maximum-likelihood phylogenies. Mol. Biol. Evol. 2015, 32, 268-274. [CrossRef] [PubMed]

29. Drozdetskiy, A.; Cole, C.; Procter, J.; Barton, G.J. JPred4: A protein secondary structure prediction server. Nucleic Acids Res. 2015, 43, W389-W394. [CrossRef]

30. Jones, D.T. Protein secondary structure prediction based on position-specific scoring matrices. J. Mol. Biol. 1999, 292 , 195-202. [CrossRef] [PubMed]

31. Yachdav, G.; Kloppmann, E.; Kajan, L.; Hecht, M.; Goldberg, T.; Hamp, T.; Honigschmid, P.; Schafferhans, A.; Roos, M.; Bernhofer, M.; et al. PredictProtein-an open resource for online prediction of protein structural and functional features. Nucleic Acids Res. 2014, 42, W337-W343. [CrossRef] [PubMed]

32. Liu, W.; Xie, Y.; Ma, J.; Luo, X.; Nie, P.; Zuo, Z.; Lahrmann, U.; Zhao, Q.; Zheng, Y.; Zhao, Y.; et al. IBS: An illustrator for the presentation and visualization of biological sequences. Bioinformatics 2015, 31, 3359-3361. [CrossRef]

33. Meng, X.; Li, C.; Bao, H.; Fang, J.; Liu, Z.; Zhang, Y. Validating the importance of two acetylcholinesterases in insecticide sensitivities by RNAi in Pardosa pseudoannulata, an important predatory enemy against several insect pests. Pest. Biochem. Physiol. 2015, 125, 26-30. [CrossRef] [PubMed]

34. Livak, K.J.; Schmittgen, T.D. Analysis of relative gene expression data using real-time quantitative PCR and the 2(-Delta Delta C(T)) method. Methods 2001, 25, 402-408. [CrossRef] [PubMed]

35. Vandesompele, J.; De Preter, K.; Pattyn, F.; Poppe, B.; Van Roy, N.; De Paepe, A.; Speleman, F. Accurate normalization of real-time quantitative RT-PCR data by geometric averaging of multiple internal control genes. Genome Biol. 2002, 3, 1-12. [CrossRef] [PubMed]

36. Swift, M.L. GraphPad prism, data analysis, and scientific graphing. J. Chem. Inf. Comput. Sci. 1997, 37, 411-412. [CrossRef] 